\title{
PESEM Proyecto Educativo Socio Empresarial una apuesta para formar, cautivar y educar al público de interés de la cooperativa del departamento del Cauca CODELCAUCA
}

\section{PESEM socio-business educational project a bet to train, captivate and educate the public of interest of the cooperative of the department of Cauca CODELCAUCA}

\author{
Mireya Realpe-Leyton ${ }^{1}$, Concepción Barreda-Ramírez ${ }^{2}$ \\ ${ }^{1}$ Grupo Ilama, Universidad Nacional Abierta y a distancia, Colombia, \\ ORCID: https://orcid.org/0000-0002-6209-5354, E-mail: mireley21@gmail.com \\ ${ }^{2}$ Grupo Ilama, Universidad Nacional Abierta y a distancia, Colombia, \\ ORCID: https://orcid.org/0000-0001-6993-3640, E-mail: concepcion.barreda@unad.edu.co
}

Cómo citar: Realpe - Leyton, M., \& Barreda - Ramírez, C. (2019). PESEM Proyecto Educativo Socio Empresarial una apuesta para formar, cautivar y educar al público de interés de la Cooperativa del Departamento del Cauca CODELCAUCA. Revista Científica Profundidad Construyendo Futuro, 10(10), 59-68. https://doi.org/10.22463/24221783.2631

Recibido: 14 de Septiembre de 2018 /Aprobado: 15 de Noviembre de 2018

\begin{abstract}
Resumen
La investigación efectuada responde a la necesidad de establecer una propuesta de Proyecto Educativo Social y Empresarial (PESEM) para la Cooperativa CODELCAUCA, entidad del sector solidario que ofrece sus servicios hace 42 años y es la Cooperativa local más grande del Departamento del Cauca, la investigación tiene como objetivo construir una propuesta de formación que fomente la cultura de la economía solidaria y mejore el sentido de pertenencia del asociado, diseñando estrategias pedagógicas pertinentes que promuevan su participación, planteando una serie de alternativas de capacitación orientadas a mejorar aspectos educativos. La metodología utilizada se enmarca dentro de un enfoque cuantitativo de tipo descriptivo, se hizo uso de herramientas de investigación como la entrevista, la interacción conversacional y la encuesta a 350 asociados; se tuvo conocimiento de la situación actual de la organización y con base en ella se realizó el PESEM para la entidad, su desarrollo permitió identificar las necesidades de formación presentes entre los asociados y demás integrantes de la Cooperativa, como resultado de los cambios socio económico que se han gestado en los últimos años, así mismo, se evidenció el importante papel que cumplen las tecnologías de información y comunicación en los procesos educativos hoy.
\end{abstract}

Palabra clave: Cooperativismo, Desarrollo, Educación, Estrategia, Solidaridad, Socio - empresarial, PESEM.

\begin{abstract}
The research carried out responds to the need to establish a proposal for a Social and Business Educational Project (PESEM) for the CODELCAUCA Cooperative, an entity in the solidarity sector that has offered its services 42 years ago and is the largest local Cooperative in the Department of Cauca, the research Its objective is to build a training proposal that fosters the culture of the solidarity economy and improves the member's sense of belonging, designing pertinent pedagogical strategies that promote their participation, proposing a series of training alternatives aimed at improving educational aspects. The methodology used is framed within a descriptive quantitative and qualitative approach, using research tools such as the interview, conversational interaction and the survey of 350 associates; The current situation of the organization was known and based on it, the PESEM was carried out for the entity, its development allowed identifying the training needs present among the members and other members of the Cooperative,
\end{abstract}


as a result of the socio-economic changes that have been developed in recent years, likewise, the important role that information and communication technologies play in educational processes is evident today.

Keyword: Cooperativism, Development, Education, Strategy, Solidarity, Socio - business, PESEM.

\section{Introducción}

El mercado globalizado y la competitividad generan en las organizaciones del sector solidario señales de alerta sobre el valor agregado que brindan a sus asociados; las empresas de hoy en día establecen planes en favor de ampliar sus posibilidades de desarrollo. Los líderes y administradores del sector deben tener en claro cuál es su razón de ser, a donde se quieren llegar y sobre qué bases se sustentan cada una de sus acciones; por eso es fundamental el conocimiento del medio tanto interno como externo en el cual influye la empresa.

El presente proyecto de investigación atiende la necesidad de diseñar una propuesta de Proyecto Educativo Social y Empresarial (PESEM) para la Cooperativa del Departamento del Cauca (CODELCAUCA). Tal como lo cita la Directiva 031 del 2000 emitida por el Ministerio de Educación y DANSOCIAL:

Toda organización solidaria debe contar con un plan de desarrollo que atienda sus necesidades reales y que potencialice la gestión de la empresa asociativa en procura de la buena marcha de la misma y del mejoramiento de la calidad de vida de los asociados y de su entorno. Este plan estará sustentado en un Proyecto Educativo Socio-Empresarial, el cual consiste en el proceso permanente que orienta la ejecución del plan de desarrollo a través de actividades educativas enmarcadas en los ámbitos de investigación, promoción, formación, capacitación y asistencia técnica. (p.1)
El Proyecto Educativo Social y Empresarial PESEM es un instrumento de apoyo de las organizaciones de economía solidaria, para orientar de manera eficaz los programas de educación. El PESEM es una herramienta que en su diagnóstico situacional contiene: un análisis del entorno, un análisis interno de sus referentes desde la perspectiva educativa y las normas que rigen para las organizaciones solidarias que imparten educación, cumplir con este proyecto permitirá potenciar las capacidades de la cooperativa y del ámbito social y laboral de los asociados. En esta investigación se identifican todos los factores para realizar este diseño.

\section{Marco teórico}

El PESEM hace parte fundamental del Plan Estratégico de CODELCAUCA, la formulación de las estrategias en favor de la consolidación y aplicación del proyecto la hacen competitiva, "La estrategia competitiva consiste en desarrollar una amplia formula de cómo la empresa va a competir, cuáles deben ser sus objetivos y qué políticas serán necesarias para alcanzar tales objetivos" (Porter, 2009, p.219).

La Planificación Estratégica es utilizada para trazar el camino que posibilite avanzar hacia estos sueños, identificando claramente las oportunidades. El concepto de Planificación Estratégica ha sido definido por varios autores. Kotler, 1990 explica que la planificación estratégica es el proceso gerencial de desarrollar y mantener una dirección estratégica que pueda alinear las 
metas y recursos de la organización con las oportunidades cambiantes del mercado, permitiendo que al identificar las mismas se dé un mejoramiento en la operación de la empresa, así como el establecimiento formal de planes o proyectos para el aprovechamiento integral de dichas oportunidades.

En el campo organizacional, sirve para formular y ejecutar las estrategias de la organización con la finalidad de insertarla, según su misión, en el contexto en el que se encuentre.

Para Drucker "La Planeación Estratégica es el proceso continuo basado en el conocimiento más amplio posible del futuro, considerando que se emplea para tomar decisiones en el presente, las cuáles implican riesgos futuros en razón de los resultados esperados" (1954, p.110)

Para este autor de la administración moderna, un aspecto que ha influido en la renovación de la nueva Gerencia es la descentralización y la delegación de poder a los empleados, la dirección por objetivos, la aplicación del management a otros campos sociales y uno de los aspectos más importantes es el enfoque de estudiar las personas en la empresa como un recurso, y no como un costo. Drucker acuñó la expresión "recursos humanos" que, a diferencia de los otros recursos, este tiene "la capacidad de coordinar, integrar, juzgar e imaginar además de autocontrolarse"

Proyecto Educativo Social y Empresarial. De acuerdo a lo establecido por la Unidad Administrativa Especial de Organizaciones Solidarias, toda organización solidaria debe preparar un proyecto educativo socio empresarial solidario (PESEM), que oriente la ejecución de actividades educativas, en los ámbitos de investigación, promoción, formación, capacitación y asistencia técnica como soporte del plan de desarrollo con el fin de alcanzar las metas sociales planteadas para los asociados, empleados, sus familias y la comunidad en general.

El PESEM parte de la identificación de las necesidades de formación de todos los miembros que conforman la organización de economía solidaria (Asociados, Directivos, Colaboradores y Comunidad en general) y se articula con la planeación y los objetivos estratégicos de la Cooperativa.

Las actividades que se planteen pueden orientarse a diversos ejes, tales como la sensibilización hacia la economía solidaria, la capacitación y la investigación, mejorando las habilidades y destrezas de todos con el fin de alcanzar los objetivos comunes.

Educación Solidaria. Es un proceso planificado y sistemático que contribuye de manera importante a una organización para fortalecer su base social (Asociados, Directivos y Colaboradores), mejorando sus capacidades y logrando así el alcance de sus objetivos organizacionales.

No obstante, cualquier proyecto de educación solidaria debe obedecer a los principios establecidos en la Ley General de la Educación, tales como:

- Percibir de manera integral al asociado como ser humano, como persona, agente social participativo y productivo para su plena realización individual y grupal.

- Estimular y generar amplios procesos de desarrollo empresarial participativo que involucren a las organizaciones solidarias en el crecimiento económico y social de la localidad, la región y el país, así como a 
PESEM proyecto educativo socio empresarial una apuesta para formar, cautivar y educar al público de interés de la cooperativa del departamento del Cauca CODELCAUCA

quienes opten por participar en el modelo Solidario.

- Desarrollar y fortalecer el modelo solidario para la generación de empleo, a través de empresas asociativas que faciliten mejorar las condiciones de vida de los asociados y comunidad en general.

- Motivar a los asociados en la gestión de su empresa asociativa. Participando en el diseño y ejecución de planes y proyectos de desarrollo que incidan en la vida social, económica, política, administrativa y cultural de la organización y su entorno.

- Incentivar la generación de conocimientos humanísticos, sociales, científicos, técnicos y gerenciales más avanzados que mediante la investigación permitan fortalecer las organizaciones solidarias.

- Promover una cultura ecológica solidaria en los asociados para garantizar un desarrollo sostenible desde las actividades socioeconómicas que ejecutan las empresas asociativas.

- Contribuir a la formación en el respeto a la Ley y a los principios que regulan a las organizaciones solidarias.

- Desarrollar el sentido de pertenencia de los asociados hacia su organización y hacia el Sector Solidario, fomentando una cultura de autocontrol sobre la gestión de las mismas.

Así mismo, la identificación de las necesidades de capacitación está relacionada con los objetivos estratégicos de la organización y el perfil de los asociados, los cuales se pueden provenir además de diversas fuentes tales como:

- Cambios del entorno: Los cambios que se presentan en los diversos entornos en los que se desenvuelve tanto la organización como los asociados, tales como el entorno económico, social, cultural, tecnológico, ambiental, traen consigo inquietudes que pueden ser abordadas desde el campo de la educación solidaria.

- Procesos educativos anteriores: Mediante actividades de formación anteriores se pueden vislumbrar otras áreas en las cuales los asociados necesiten refuerzo o capacitación, tanto en lo relacionado con sus funciones dentro de la Cooperativa, como con las competencias en su área de desempeño profesional.

- Quejas y reclamos presentadas por los asociados: En cualquier organización, son una fuente de crecimiento y mejora continua, por ende, son además un poderoso recurso de información con el fin de identificar falencias de la Cooperativa y de cómo enfrentarlas, en este caso a través de una herramienta asertiva como lo es la educación.

Legislación: En lo concerniente a la educación solidaria, existe una serie de reglamentación, normas e instrucciones que deben tenerse en cuenta la hora de elaborar sus planes y la manera en que estos inciden en los recursos.

\section{Metodología}

La población objeto de estudio fue los 3.818 asociados de la Cooperativa; para el cálculo de la muestra se aplicó la fórmula para poblaciones finitas, obteniendo una muestra de 350 asociados. La naturaleza del estudio se enmarcó dentro del enfoque cuantitativo de tipo descriptivo, se utilizó como instrumento un cuestionario estructurado de 17 preguntas las cuales fueron direccionadas con el fin de identificar las necesidades de formación, capacitación y educación de los asociados. Fue aplicada en los eventos culturales, de recreación y de capacitación que programó la entidad en todo el Departamento del Cauca; una vez recolectada la información se 
procedió a tabular los datos obtenidos, graficarlos y analizarlos.

Adicionalmente se realizó la revisión de diferentes documentos, fuentes bibliográficas, normatividad, monografías especializadas, revistas, bases de datos, informes y publicaciones para corroborar y analizar cifras, ideas y planteamientos logrados.

El proceso investigativo desarrolló en las siguientes fases:

- Revisión bibliográfica. En esta fase se estudiaron las investigaciones, normatividad y los textos relacionados sobre el tema objeto de análisis y los hallazgos más relevantes en la materia realizados hasta la fecha.

- Trabajo de campo. Se desarrolló una exploración que incluye encuestas a los asociados, colaboradores y demás participantes de la cooperativa para conocer sus expectativas de formación de acuerdo al Proyecto Educativo Socio-empresarial de CODELCAUCA.

- Análisis y procesamiento de datos. En esta fase se tomaron medidas de las diferentes variables para el análisis de los resultados del trabajo de campo como son niveles académicos, ingresos socioeconómicos, alternativas de formación, entre otras. Así como de aquellas otras variables que a raíz de la fase de exploración cualitativa surjan y sea adecuado incluir. Se plantearán posteriormente la definición del Proyecto Educativo Socio-empresarial y conclusiones de la investigación.

- A través de la investigación observacional se recopilaron datos primarios a partir de la observación de personas, acciones y situaciones pertinentes. Por ejemplo, efectuar visitas a las tres agencias de la Cooperativa, para observar cómo funciona la entidad, su planta física, y los procesos que manejan.
- Un insumo importante para establecer las estrategias pedagógicas fue el contacto directo con los encuestados, ya que complementaban el instrumento y concedían información adicional relevante para la investigación, especialmente los encuestados con cargos directivos.

- Aplicación de una Matriz DOFA para identificar los aspectos internos y externos de la Cooperativa y la manera de integrar estos resultados en el PESEM.

\section{Resultados}

Por cada uno de los ejes temáticos consultados se presentan los tres puntajes más altos en cada categoría lo anterior es información base para plantear los programas de formación teniendo en cuenta los gustos, preferencias y necesidades de los asociados y su núcleo familiar.

Respecto a las áreas de cooperativismo en las que les gustaría capacitarse se percibe que el interés radica en conocer a profundidad el funcionamiento de la Cooperativa (53\%), siendo que los temas que destaca la encuesta son: estatutos y reglamentos, el control social $(18 \%)$ y los principios y valores (13\%).

Cabe destacar que, siendo la mayoría de los asociados empleados, desean recibir capacitación en creación de empresas (50\%), administración (16\%) y finanzas (13\%); lo que deja entrever un gran interés de los asociados por mejorar su nivel de ingresos $y$ por ende su calidad de vida.

Con relación a la formación en sistemas el resultado de esta pregunta está asociado con el aspecto anterior, ya que revela el interés de los asociados por capacitarse en un área que puede ser importante para la creación de nuevas unidades empresariales como los 
PESEM proyecto educativo socio empresarial una apuesta para formar, cautivar y educar al público de interés de la cooperativa del departamento del Cauca CODELCAUCA

sistemas: mantenimiento computadores (47\%), hoja electrónica (24\%) y uso de internet $(20 \%)$

En cuanto al aprendizaje de idiomas, se ratifica la importancia del inglés (57\%) como idioma clave para la interacción global, en las esferas económica, cultural y social, siendo el ítem más llamativo entre las respuestas dadas, seguido de francés $(25 \%)$ y portugués $(16 \%)$.

En cuanto a otras áreas de formación encontramos la gastronomía (60\%) como principal respuesta, teniendo en cuenta las actuales tendencias de consumo mundial, que han puesto a la gastronomía como una de las áreas más demandadas en la Academia en los últimos años. Se debe tener en cuenta que en la ciudad de Popayán se realiza el Congreso Gastronómico desde hace varios años, con su consecuente impacto en la sociedad, lo que puede haber influido un poco en la respuesta.

En cuanto a las manualidades se destacan en los resultados la inclinación de los asociados hacia áreas que puedan implicar una nueva fuente de ingresos, como la decoración con bombas (38\%), florales (27\%) y navideños $(28 \%)$.

Las prácticas deportivas también están presentes en los intereses de formación de los asociados. Aparecen con gran importancia dos de los deportes más tradicionales: fútbol, básquetbol y natación. Este aspecto es importante, pues debe tenerse en cuenta que los procesos de formación deben ser integrales.

En cuanto a la formación relacionada con el medio ambiente se detecta una motivación hacia el manejo o reutilización del material reciclado que bien enfocado puede generar alternativas empresariales.
Un aspecto en definitivo que marca la formación personal están los temas relacionados con crecimiento personal (43\%), aspecto que se aplica a todos los asociados y su núcleo familiar, la estrategia para que accedan es generar alternativas de formación por edades para motivar un espíritu positivo y adaptable que promueva su superación personal y de quienes están a su alrededor.

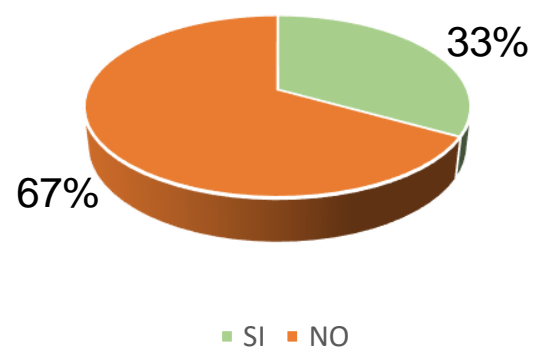

Figura 1. Ha realizado procesos de investigación

El 33\% de los asociados ha realizado procesos de investigación, la indagación sobre este aspecto permite identificar uno de los ámbitos de la educación solidaria propuesto por la Directiva 31 del 2.000 elaborado por DANSOCIAL y hace relación a la investigación que permite ejecutar acciones tendientes a la generación o producción de nuevos conocimientos y tecnologías para la proyección de las organizaciones solidarias.

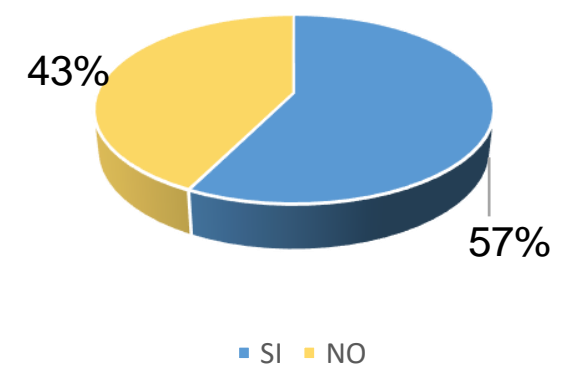

Figura 2. Fácil acceso a un computador con internet 
El 57\% de los asociados tienen acceso a un computador con internet este factor representa para la cooperativa una valiosa oportunidad de llegar a los asociados y su núcleo familiar con propuestas de educación virtual, favoreciendo la cobertura y la demanda de los programas que se contemplen en el PESEM.

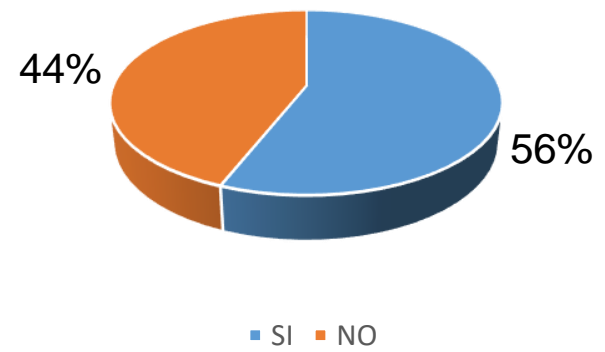

Figura 3. Fácil acceso a un celular con internet

El 56\% de los asociados pueden acceder a un celular con internet situación que favorece la estrategia de difusión y comunicación de los programas a ofrecer, además permite que los asociados por este medio puedan acceder a las capacitaciones.

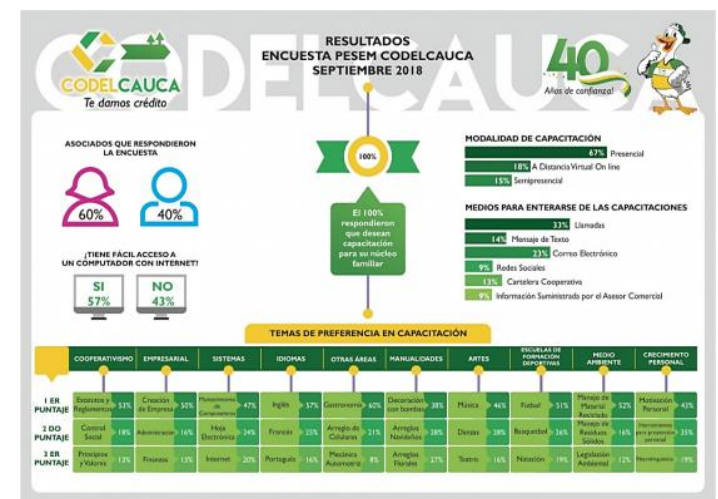

Figura 4. Infografía resultados encuesta PESEM

CODELCAUCA. Fuente: Elaboración propia.

Con la información obtenida se construyó el Proyecto Educativo Socio Empresarial de la entidad teniendo en cuenta que las necesidades propias de formación en CODELCAUCA parten de la identificación realizada a través de la encuesta y el diagnóstico interno y externo de la organización, en los siguientes componentes:

-Primer Componente: Sensibilización en cooperativismo y economía solidaria.

-Segundo Componente: Desarrollo de competencias del asociado, directivo y colaborador de la Cooperativa.

-Tercer Componente: Emprendimiento Empresarial e investigación.

-Cuarto Componente: Formación integral.

Sobre los cuatro componentes se han establecido las competencias que buscan fortalecer los procesos de capacitación y formación a fin de transferir lo aprendido en su trabajo, en su familia y en su proyecto empresarial, "una competencia es una capacidad para movilizar diversos recursos cognitivos para hacer frente a un tipo de situaciones (Perrenoud, 2000), es ser capaz de transferir lo aprendido, de tener autonomía en el aprendizaje y de resolver problemas.

Tabla 1. Categorías de las competencias por cada uno de los cuatro componentes

\begin{tabular}{|c|c|}
\hline COMPONENTE & COMPETENCIAS \\
\hline Primer Componente: & \\
\hline Sensibilización en & \\
\hline cooperativismo & Conciencia \\
\hline economía solidaria. & Organizacional, \\
\hline Categoría & desarrollo de relaciones, \\
\hline Competencia: & impacto e influencia \\
\hline IMPACTO & \\
\hline INFLUENCIA & \\
\hline Segundo Componente: & \\
\hline Desarrollo de & Adminictromión \\
\hline asociado, directivo y & recursos, Búsqueda de \\
\hline $\begin{array}{l}\text { colaborador de la } \\
\text { Cooperativa. }\end{array}$ & información, Iniciativa, \\
\hline Categoría & Planificación y \\
\hline Competencia: & organización del trabajo, \\
\hline ACCIÓN & Toma de decisiones \\
\hline OBTENCIÓN & \\
\hline RESULTADOS & \\
\hline
\end{tabular}




\begin{tabular}{|c|c|}
\hline Tercer Componente: & \\
\hline Emprendimiento & Análisis numérico, \\
\hline $\begin{array}{l}\text { Empresarial } \\
\text { investigación }\end{array}$ & $\begin{array}{l}\text { Análisis y resolución de } \\
\text { problemas, Pensamiento }\end{array}$ \\
\hline Categoría & analítico, Pensamiento \\
\hline encia: & \\
\hline TIVO & \\
\hline Cuarto Componente: & \\
\hline Formación integral & ianza, \\
\hline Categoría & \\
\hline Compe & Disponibilidad, \\
\hline $\mathrm{EF}$ & Identificación \\
\hline PERSOI & \\
\hline
\end{tabular}

Fuente: Autores de la investigación.

Primer Componente: Sensibilización en cooperativismo y economía solidaria. En este eje se busca dar a conocer la filosofía, principios y finalidad del modelo solidario y la manera en que las organizaciones cooperativas se diferencian de las demás. A través de la ejecución de este componente se busca desarrollar y fomentar la cultura hacia el sector solidario, a través de actividades de formación con el fin de generar actitudes y conductas en favor del crecimiento y continuidad del modelo solidario, la competencia de este componente es el impacto e influencia; Segundo Componente: Desarrollo de competencias del asociado, directivo y colaborador de la Cooperativa. En este componente se busca fortalecer las diferentes competencias de los asociados en los roles que ejerce dentro de la Cooperativa. El grupo de competencias tiene relación a la acción y obtención de resultados.

Categoría Competencia: acción y obtención de resultados. La esencia de esta categoría es la orientación hacia la acción, dirigida más al cumplimiento de las tareas que a atender a las personas; Tercer Componente: Emprendimiento Empresarial e investigación. Otro de los ejes que se ha identificado a través de las encuestas que representa gran interés para los asociados es lo relacionado con el mundo empresarial. En esta competencia se encuentran las competencias de tipo cognitivo; Cuarto Componente: Formación integral. Los programas de capacitación deben estar orientados a formar más que en habilidades intelectuales, sino en la totalidad del ser humano, estimulando habilidades sociales $\mathrm{y}$ emocionales.

La idea de esta dimensión es fortalecer la carga axiológica de las personas para que expresen esos valores en la vida cotidiana, de manera que los asociados puedan llegar a convertirse en ejemplos a seguir y además estimulen a los más jóvenes.

Los valores éticos son fundamentales para lograr una buena convivencia, lo cual puede ayudar en los procesos de aprendizaje. La formación integral está orientada a garantizar el desenvolvimiento armónico y fluido de las personas en diferentes contextos, de manera que sean capaces de tomar decisiones, trabajar en equipo y expresar sus ideas con convicción. Desarrollar este tipo de comportamientos puede desembocar fácilmente en un mayor bienestar y una mejor calidad de vida y convivencia social. Las competencias para esta categoría están relacionadas con la efectividad personal.

\section{Discusiones}

sector solidario, son cuestión de toda la comunidad de una región, que lo requiere con urgente necesidad. Entiéndase por Educación Solidaria el proceso permanente orientado a fortalecer la práctica de valores de solidaridad, cooperación y ayuda mutua, con el fin de generar actitudes y conductas que permitan consolidar un compromiso social frente a la misión que deben cumplir las Organizaciones Solidarias de conformidad 
con las previsiones de la Ley 454/98. Tener un proyecto educativo de acuerdo a esas necesidades permite en Cooperativas grandes como CODELCAUCA, cumplir su misión y llevar a las familias de asociados lo que realmente necesitan.

En armonía con lo anterior, la Educación Solidaria busca consolidar procesos de autogestión empresarial en función del desarrollo sostenible de la calidad de vida de los asociados y de la comunidad en general.

La educación que deben adelantar las organizaciones solidarias tendrá como sujetos: el comité de educación, los asociados, los órganos de administración, de vigilancia y control, los empleados, las personas naturales o jurídicas que imparten educación a las organizaciones solidarias, los organismos de Integración del sector y las entidades gubernamentales y no gubernamentales que coadyuven al logro de los fines del Proyecto Educativo Social y Empresarial de la organización, los cuales conforman la comunidad educativa solidaria y según su competencia, participarán en el diseño, ejecución y evaluación de los Proyectos Educativos Sociales y Empresariales y en la buena marcha de la empresa asociativa

\section{Conclusiones}

El desarrollo de la propuesta del PESEM para CODELCAUCA permitió identificar las necesidades de formación presentes entre los asociados y demás integrantes de la Cooperativa, como resultado de los cambios socio económico que se han gestado en los últimos años.

Así mismo, se evidenció el importante papel que cumplen las tecnologías de información y comunicación en los procesos educativos de hoy, lo cual es sin duda un condicionante para los objetivos del PESEM.

Un PESEM adecuadamente desarrollado ayudará a la Cooperativa a fortalecer los lazos con los asociados, mejorar la sensibilidad respecto al sector solidario y consolidar su presencia en el Departamento del Cauca.

El PESEM debe fortalecerse a partir de las necesidades de los asociados, quienes son el público objetivo primordial para las actividades de formación, por lo tanto, consultar sus expectativas frente al programa fue un insumo vital para el desarrollo de la propuesta aquí consignada.

El PESEM debe reflejar la integralidad tanto de las necesidades de capacitación de los asociados, como de sus competencias personales y expectativas de crecimiento profesional y económico tal como lo cita la Directiva 031 del 2.000

La implementación del PESEM requiere de la integración de la Cooperativa con instituciones educativas y otros actores que puedan ayudar a que los objetivos aquí planteados se hagan realidad.

El PESEM establece las orientaciones generales para contribuir al mejoramiento de la formación de asociados, directivos y colaboradores, con el fin de lograr un perfil acorde a una organización del sector solidario.

\section{Referencias}

Decreto 4122 de 2011. "por medio del cual se transforma el Departamento Administrativo Nacional de Economía Solidaria, Dansocial, en la Unidad. 
PESEM proyecto educativo socio empresarial una apuesta para formar, cautivar y educar al público de interés de la cooperativa del departamento del Cauca CODELCAUCA

Departamento A (2000). Directiva 31 de 7 de julio. DANSOCIAL. Administrativo Nacional de Economía Solidaria.

Drucker. (1954). The Practice of Management

Kotler. P. (1990). Introducción al marketing.

Ley 454 de 1998. Por la cual se determina el marco conceptual que regula la economía solidaria, se transforma el Departamento Administrativo Nacional de Cooperativas en el Departamento Nacional de la Economía Solidaria, se crea la Superintendencia de la Economía Solidaria, se crea el Fondo de Garantías para las Cooperativas Financieras y de Ahorro y Crédito, se dictan normas sobre la actividad financiera de las entidades de naturaleza cooperativa y se expiden otras. Agosto 6 de 1998. D.O.N ${ }^{\circ}$ 43.357,

Ley 115 de 1994. Como Ley General de la Educación en Colombia, fija los parámetros para los procesos de formación, su calidad, cubrimiento y fines.

Ley 79 de 1988 "Por la cual se actualiza la Legislación Cooperativa".

Perrenoud. (2000). La estrategia de proyectos. Aprender en la escuela a través de proyectos: ¿Por qué? ¿Cómo?" Revista de Tecnología Educativa (Santiago de Chile), 14 (3), pp. 311-321.

Porter. M. E. (2009). Ser competitivo. Ediciones Deusto. Barcelona.
Resolución 194 de 2001 de DANSOCIAL: "por la cual se establecen los fundamentos, criterios, parámetros y requisitos de los procesos de inducción y educación en economía solidaria, y la expedición de los certificados de acreditación sobre educación en teoría y práctica de la misma" 